\title{
Logarithmic-Sobolev and multilinear Hölder's inequalities via heat flow monotonicity formulas
}

\author{
A. Abolarinwa*, N.K. Oladejo, S.O. Salawu, C.A. Onate \\ Department of Physical Sciences, Landmark University, P. M. B. 1001, Omu-Aran, Kwara State, Nigeria
}

\section{A R T I C L E I N F O}

\section{Article history:}

Received 8 March 2019

Revised 5 July 2019

Accepted 29 July 2019

Available online 9 August 2019

\section{MSC:}

26A48

$35 \mathrm{~K} 05$

52A40

\section{Keywords:}

Entropy

Heat flow

Sobolev inequality

Hölder inequality

Gaussian measure

\begin{abstract}
A B S T R A C T
Heat flow monotonicity formulas have evolved in recent years as a powerful tool in deriving functional and geometric inequalities which are in turn useful in mathematical analysis and applications. This paper aims mainly at proving Logarithmic Sobolev and multilinear Hölder's inequalities through the heat flow method. Precisely, two entropy monotonicity formulas are constructed via the heat flow. It is shown that the first entropy monotonicity formula is intimately related to the concavity of the power of Shannon entropy and Fisher Information, from which the associated logarithmic Sobolev inequality for probability measure in Euclidean setting is recovered. The second monotonicity formula combines very well with convolution and diffusion semigroup properties of the heat kernel to establish the proof of the multilinear Hölder inequalities.
\end{abstract}

(C) 2019 Elsevier Inc. All rights reserved.

\section{Introduction and preliminaries}

\subsection{Introduction}

Heat flows and entropy formulas are two powerful analytic tools widely used in various settings of mathematical analysis and scientific research. Heat flow, though classical, has attracted more attention in proving functional inequalities $[2,4,6]$ since the work of Carlen, Loss and Lieb [10]. Their work [10] was motivated by statistical mechanical considerations, and was used to derive some sharp inequalities on the sphere. Entropy formulas were used in the 70's to prove some embedding and hypercontractive estimates [16,17], consequently leading to Sobolev-type inequalities. Since the complete proof of Poincare conjecture by Perelman [22], many entropy monotonicity formulas have been proved [2,3,21,25,26] and thereby leading to several functional inequalities such as Harnack and Logarithmic Sobolev inequalities. A motivation for this is that applications of these inequalities are ubiquitous in various fields such as PDEs, Euclidean analysis, harmonic analysis, information theory, optimal transport, kinetic theory and so on (cf. $[4,11,18,19,26]$ ).

This paper mainly aims at proving two important inequalities in mathematical analysis and applications, namely; Logarithmic Sobolev and multilinear Hölder's inequalities. In order to achieve this, two entropy monotonicity formulas are constructed and their monotonicity proved by the method of the heat flow. The first entropy monotonicity formula is shown to

\footnotetext{
* Corresponding author.

E-mail address: abolarinwa.abimbola@lmu.edu.ng (A. Abolarinwa).
} 
be intimately related to the concavity of the power of Shannon entropy and Fisher Information [12,23,24], from which the associated logarithmic Sobolev inequality for probability measure in Euclidean setting is recovered. The second monotonicity formula is combined with convolution and diffusion semigroup properties of the heat kernel to establish the proof of the multilinear Hölder inequalities. This approach, that is combining heat flow and entropy formula, appeared more simplified than using either heat flow or entropy formula independently. The results obtained here can be compared with existing ones $[5,11,18,25,26]$. Another motivation for this work is that these inequalities can be easily seen as the consequence of the ability of functionals involving powers of smooth solution to the heat equation to approach their extremal values as time grows infinitely.

\subsection{Preliminaries}

This paper is concerned with the monotonicity formulas for the heat equation on Euclidean space, $\mathbb{R}^{n}$, and their geometric consequences for some functional inequalities, namely, Gaussian logarithmic Sobolev inequality introduced by L. Gross in [16] and the well known multilinear Hölder inequalities. The heat equation

$$
\left(\frac{\partial}{\partial t}-\Delta\right) u(t, x)=0
$$

with its positive solution $u=u(t, x)$, where $\frac{\partial}{\partial t}$ is the partial derivative with respect to $t, \Delta=\sum_{j=1}^{n} \frac{\partial^{2}}{\partial x_{j}^{2}}$ and $x=\left(x_{1}, \ldots, x_{n}\right) \in$ $\mathbb{R}^{n}$ is considered. The standard Gauss-Weierstrass heat kernel in $\mathbb{R}^{n}$ is given by $K(t, x, y)=(4 \pi t)^{-\frac{n}{2}} e^{-\frac{|x-y|^{2}}{4 t}}$. It is well known that the convolution of $K$ with $u(0, x)=u(x)$ solves the heat Eq. (1.1) and possesses important properties which include smoothness, positivity and diffusion semigroup (see [15] for an example). These properties are of paramount importance in this work. Now, set the solution of (1.1) to be a Gaussian density function (see [9] for details about Gaussian functions) with respect to the Lebesgue measure on $\mathbb{R}^{n}$,

$$
u(t, x):=(4 \pi \tau)^{-\frac{n}{2}} e^{-f(t, x)}, \quad \tau=\tau(t)>0, \quad f: \mathbb{R}^{n} \rightarrow(0, \infty)
$$

with normalization $\int_{\mathbb{R}^{n}} u d \mu=1$ and $\frac{\partial \tau}{\partial t}=1$, where $d \mu=(2 \pi)^{-\frac{n}{2}} e^{-\frac{|x|^{2}}{2}} d x$ is the Gaussian measure on $\mathbb{R}^{n}$ and $d x$ is the Lebesque measure. Then, by direct calculation we obtain

$$
\left\{\begin{array}{l}
f=-\ln u-\frac{n}{2}(4 \pi \tau) \\
\frac{\partial u}{\partial t}=\left(-\frac{n}{2 \tau}-\frac{\partial f}{\partial t}\right) u \\
\Delta u=\left(-\Delta f+|\nabla f|^{2}\right) u
\end{array}\right.
$$

This implies that the problem can be reduced to the study of the coupled system

$$
\left\{\begin{array}{l}
\frac{\partial u}{\partial t}=\Delta u \\
\frac{\partial f}{\partial t}=\Delta f-|\nabla f|^{2}-\frac{n}{2 \tau} \\
\frac{\partial \tau}{\partial t}=1 \\
u(0, x)=u(x) .
\end{array}\right.
$$

So, in a similar spirit to Perelman $\mathcal{W}$-entropy, we define a new entropy functional

$$
W(\tau, f)=\int_{\mathbb{R}^{n}}\left(\tau|\nabla f|^{2}+f-n\right)(4 \pi \tau)^{-\frac{n}{2}} e^{-f} d x
$$

with $\int_{\mathbb{R}^{n}}(4 \pi \tau)^{-\frac{n}{2}} e^{-f} d x=1$.

Recall that Perelman $\mathcal{W}$-entropy in [22] is monotone nondecreasing in time under the Ricci flow coupled with nonlinear conjugate heat equation and combines very well with Ricci soliton metric on compact manifold, where its monotonicity implies that indeed a shrinking breather is a gradient shrinking soliton. Gradient shrinking solitons arise as a singularity models for Ricci flow. The Euclidean space $\mathbb{R}^{n}$ is readily an example while round cylinder $\mathbb{S}^{n-k} \times \mathbb{R}^{k}$ or its quotient provides another class of examples of gradient shrinking solitons. After renormalization, Perelman's entropy vanishes on Euclidean spaces. It can also be used to establish condition for isometry between a nonnegative Ricci curvature manifold and Euclidean space (see also [1,21]). With nonnegativity condition on the Ricci curvature, Perelman's entropy may also imply Li-Yau gradient estimate $[19,21,28]$

$$
\tau(\Delta \ln u) \leq \frac{n}{2}
$$


which may in turn imply Nash inequality viz a viz isoperimetric inequality. This is in support of classical fact that classical inequalities of Faber-Krahn, Sobolev, Log-Sobolev, Nash, Isoperimetric and Heat Kernel upper bound inequalities are indeed equivalent on any Riemannian Manifolds as well as Euclidean Spaces, (see the following references for instance $[1,13,14,28]$ ).

This paper proves that entropy (1.4) is monotonically nonincreasing in time and shows that the resulting monotonicity formula is strongly related to classical entropies of Shannon and Fisher information. The entropies of Shannon and Fisher information for heat equation are respectively defined as

$$
H(u)=-\int_{\mathbb{R}^{n}} u \ln u d x \quad \text { and } \quad I(u)=\int_{\mathbb{R}^{n}}|\nabla \ln u|^{2} u d x .
$$

The entropy $H(u)$ was first introduced in Shannon [23]. They have numerous applications in information theory, combinatorics, thermodynamics and statistical mechanics. For instance $H(u)$ can be used to provide alternative proof of LoomisWhitney inequality [20]. Note that Loomis-Whitney inequality is a natural generalization of multilinear Hölder inequality [7]. It also provides a very useful inequality, namely Shannon entropy power inequality.

$$
e^{\frac{2}{n} H(X+Y)} \geq e^{\frac{2}{n} H(X)}+e^{\frac{2}{n} H(Y)}
$$

where $X$ and $Y$ are independent random variables with probability density function in $L^{2}\left(\mathbb{R}^{n}\right)$ for $1<p<\infty$. See the proof of 1.5 in $[8,12,23,24]$. Most of these proofs use monotonicity property of the heat flow.

Proposition 1.1. Let $u$ be a positive solution to the heat Eq. (1.1). Then

$$
\frac{d H(u)}{d t}=I(u)
$$

and

$$
\frac{d^{2} H(u)}{d t^{2}}=-2 J(u)
$$

where $J(u)=\int_{\mathbb{R}^{n}}\left|\nabla_{i} \nabla_{j} \ln u\right|^{2} u d x$ and $\nabla_{i}$ is the gradient operator on the ith coordinate.

The proof of Proposition 1.1 is contained in Lemma 3.1 below. The relationship in the above proposition is known in literature as DeBruijin's identities and have been coupled to prove the concavity of Shannon entropy power. The concavity of Shannon entropy power asserts that

$$
\frac{d^{2} N\left(K_{t} * g\right)}{d t^{2}} \leq 0
$$

where $N(u)=\frac{1}{2 \pi e} \exp \left(\frac{2}{n} H(u)\right)$ is the entropy power and $g$ is a positive solution of the heat equation while $K_{t}^{*} g$ is the convolution of $g$ and the heat kernel. The concavity of entropy power can be viewed as being equivalent to the inequality

$$
J(u) \geq \frac{1}{n} I^{2},
$$

where

$$
-2 J(u)=\frac{d}{d t} I(u)
$$

Various proofs of this inequality are presented in literature, e.g., [12,25-27].

In [26], Toscani remarks that subsequent derivatives of Shannon entropy alternate in sign. He also considered scaling properties of Shannon entropy and Fisher Information, respectively as

$$
H(u)=H(u)-n \ln a \quad \text { and } \quad I(u)=a^{2} I(u)
$$

using scaling factor $\mathrm{g}\left(u_{a}\right) \rightarrow \mathrm{g}_{a}(v)=a^{2} \mathrm{~g}(a v), \quad a>0$, which preserves the total mass of the function $\mathrm{g}$. A more direct tool is employed to dilate these quantities and the scalings have direct consequences on the monotone property of the quantity $W$ in (1.4). All these are discussed in Section (3).

\section{Statement of results}

The main results of this paper are stated in this section. The first result concerning the monotonicity of $W=W(\tau, f)$ entropy is the following:

Theorem 2.1. Let $u$ be a positive solution of the heat Eq. (1.1) with $\int_{\mathbb{R}^{n}} u d \mu=1, \partial_{t} f=\Delta f-|\nabla f|^{2}-\frac{n}{2 \tau}, \quad \tau>0$ and $\frac{d \tau}{d t}=1$. Then

$$
\frac{d W}{d t} \leq-\frac{2 \tau}{n} \int_{\mathbb{R}^{n}}\left(|\nabla \ln u|^{2}-\frac{n}{2 \tau}\right)^{2} u d \mu
$$

The monotonicity formula is sharp, indeed, equality holds in (2.1) if $u$ should be taken to be the fundamental solution. This is verifiable by using $f(t, x)=\frac{|x|^{2}}{4 t}$. The corresponding differential Harnack inequality for the positive solution to 
the heat equation can also be obtained from this. More importantly, it is used here to derive a sharp logarithmic Sobolev inequality due to Gross [16], see also [17]. Gross log-Sobolev inequality states that

$$
\int_{\mathbb{R}^{n}} \phi^{p} \ln |\phi| d \mu \leq \int_{\mathbb{R}^{n}}|\nabla \phi|^{p} d \mu+\left(\int_{\mathbb{R}^{n}} \phi^{p} d \mu\right) \ln \left(\|\phi\|_{p}\right)
$$

for $\phi \in L^{p}\left(\mathbb{R}^{n}, d \mu\right)$ such that $|\nabla \phi| \in L^{p}\left(\mathbb{R}^{n}, d \mu\right), 1<p<\infty$, and $d \mu$ be Gaussian measure on $\mathbb{R}^{n}$. Note that there is equality if and only if $\phi$ is a constant and no constant depending on $n$ is involved. Suppose $p=2$ and $\phi$ is chosen in such a way that $\|\phi\|_{2}=1$, the inequality in (2.4) becomes

$$
\int_{\mathbb{R}^{n}} \phi^{2} \ln |\phi| d \mu \leq \int_{\mathbb{R}^{n}}|\nabla \phi|^{2} d \mu .
$$

The result is the following sharp inequality.

Theorem 2.2. Let $\phi \in L^{2}\left(\mathbb{R}^{n}, d \mu\right)$ such that $|\nabla \phi| \in L^{2}\left(\mathbb{R}^{n}, d \mu\right)$. Then

$$
\int_{\mathbb{R}^{n}} \phi^{2} \ln |\phi| d \mu \leq \frac{\varepsilon}{2 \pi} \int_{\mathbb{R}^{n}}|\nabla \phi|^{2} d \mu+C(\varepsilon, n),
$$

where $\varepsilon>0$ and $C(\varepsilon, n)$ is a constant depending on on $\varepsilon$ and $n$.

Heat flow monotonicity has turned out to be a powerful idea to proving sharp inequalities, such as Young inequality for convolution, generalized Hölder's inequality, Brascamp-Lieb inequality. For detail see for instance $[4,6,10]$. In summary, the idea is to rely on the smoothing and Markovian properties of the fundamental solution of the heat equation, which will diffuse initial solution to optimizer in a monotonic way. In a similar vein the second entropy functional is constructed as follows

$$
\Phi(t)=\int_{\mathbb{R}^{n}} \prod_{j=1}^{m} f_{j}(t, x)^{\frac{1}{p_{j}}} d \mu(x)
$$

for all nonnegative functions $f_{j} \in L^{p_{j}}\left(\mathbb{R}^{n}\right)$. Let $f_{j}$ be a solution to the heat equation, the entropy (2.5) is known to be differentiable and smoothly continuous by the smoothing properties of the heat kernel semigroup for all $t>0$. The monotonicity formula for $\Phi(t)$ is used to prove the following theorem.

Theorem 2.3. Let the product of $m$-functions $f_{j}$ be $\prod_{j=1}^{m} f_{j}(x), m \in \mathbb{N}$ and $1 \leq j \leq m$. For each $1 \leq p_{j} \leq \infty$, we have the inequality

$$
\int_{\mathbb{R}^{n}} \prod_{j=1}^{m} f_{j}(x) d \mu(x) \leq \prod_{j=1}^{m}\left\|f_{j}(x)\right\|_{L^{p_{j}}}
$$

for all nonnegative functions $f_{j} \in L^{p_{j}}\left(\mathbb{R}^{n}\right)$ with $\sum_{j=1}^{m} p_{j}^{-1}=1$.

The detail of the proof (2.6) is reserved to the last section.

\section{Proof of main result about $W(\tau, f)$}

This section is devoted to the proof of the monotonicity formula for $W(\tau, f)$. The relationship between the monotonicity formula and the classical entropy of Shannon $H$ and Fisher information $I$ are also highlighted.

\subsection{Monotonicity of $\mathrm{W}(\tau, \mathrm{f})$}

Firstly, an important lemma that will be applied in the proof of Theorem 2.1 is stated and proved. The lemma also contains the proof of Proposition 1.1.

Lemma 3.1. Let $u$ be a Gaussian density function satisfying the heat Eq. (1.1). Then

$$
\frac{\partial}{\partial t}\left(\int_{\mathbb{R}^{n}} u \ln u d \mu\right)=-\int_{\mathbb{R}^{n}}|\nabla \ln u|^{2} u d \mu
$$

and

$$
\frac{\partial}{\partial t}\left(\int_{\mathbb{R}^{n}}|\nabla \ln u|^{2} u d \mu\right)=-2 \int_{\mathbb{R}^{n}}|\nabla \nabla \ln u|^{2} u d \mu .
$$

Proof of Theorem 3.1. By direct calculation

$$
\begin{aligned}
\frac{\partial}{\partial t}\left(\int_{\mathbb{R}^{n}} u \ln u d \mu\right) & =\int_{\mathbb{R}^{n}}\left[\frac{\partial u}{\partial t} \ln u+u \frac{\partial}{\partial t}(\ln u)\right] d \mu=\int_{\mathbb{R}^{n}}\left[\Delta u \ln u+u \frac{\Delta u}{u}\right] d \mu \\
& =\int_{\mathbb{R}^{n}} \Delta u \ln u d \mu=-\int_{\mathbb{R}^{n}} \nabla u \nabla \ln u d \mu=-\int_{\mathbb{R}^{n}}|\nabla \ln u|^{2} u d \mu .
\end{aligned}
$$


This proves (3.1).

$$
\begin{aligned}
\frac{\partial}{\partial t}\left(|\nabla \ln u|^{2} u d \mu\right)= & \int_{\mathbb{R}^{n}}\left[\frac{\partial}{\partial t}\left(|\nabla \ln u|^{2}\right) u+|\nabla \ln u|^{2} \frac{\partial u}{\partial t}\right] d \mu \\
= & \int_{\mathbb{R}^{n}} 2 u \nabla \ln u \nabla\left(\frac{\Delta u}{u}\right) d \mu+\int_{\mathbb{R}^{n}}|\nabla \ln u|^{2} \Delta u d \mu \\
= & 2 \int_{\mathbb{R}^{n}} u \nabla \ln u \nabla\left(\Delta \ln u+|\nabla \ln u|^{2}\right) d \mu+\int_{\mathbb{R}^{n}} \Delta|\nabla \ln u|^{2} u d \mu \\
= & 2 \int_{\mathbb{R}^{n}} u \nabla \ln u \nabla \Delta \ln u d \mu-2 \int_{\mathbb{R}^{n}} u \Delta|\nabla \ln u|^{2} d \mu \\
& +\int_{\mathbb{R}^{n}} \Delta|\nabla \ln u|^{2} u d \mu \\
= & \int_{\mathbb{R}^{n}}\left[2\langle\nabla \ln u, \nabla \Delta \ln u\rangle-\Delta|\nabla \ln u|^{2}\right] u d \mu \\
= & -2 \int_{\mathbb{R}^{n}}|\nabla \nabla(\ln u)|^{2} u d \mu .
\end{aligned}
$$

The last equality follows from the following Bochner identity on $\mathbb{R}^{n}$

$$
\frac{1}{2} \Delta|\nabla f|^{2}=|\nabla \nabla f|^{2}+\langle\nabla f, \nabla \Delta f\rangle
$$

for every $f \in C^{\infty}$. This completes the proof of (3.2).

The proof of Theorem 2.1 is now stated.

Proof of Theorem 2.1. Using (1.2) we can rewrite the quantity (1.4) as

$$
\begin{aligned}
W & =\int_{\mathbb{R}^{n}}\left[\tau \frac{|\nabla u|^{2}}{u^{2}}-\ln u-\frac{n}{2} \ln (4 \pi \tau)-n\right] u d \mu \\
& =\int_{\mathbb{R}^{n}} \tau|\nabla \ln u|^{2} u d \mu-\int_{\mathbb{R}^{n}} \ln (u) u d \mu-\frac{n}{2} \ln (4 \pi \tau)-n \\
& =\tau I(u)+H(u)-\frac{n}{2} \ln (4 \pi \tau)-n .
\end{aligned}
$$

Combining Lemma 3.1 and Proposition 1.1, the time derivative of $W$ is obtained as follows:

$$
\begin{aligned}
\frac{d W}{d t} & =\tau \frac{d I}{d t}+I+\frac{d H}{d t}-\frac{n}{2 \tau} \\
& =\tau \frac{d I}{d t}+2 I-\frac{n}{2 \tau} \\
& =-2 \tau J+2 I-\frac{n}{2 \tau} \\
& \leq-\frac{2}{n} \tau I^{2}+2 I-\frac{n}{2 \tau}=-\frac{2 \tau}{n}\left(I-\frac{n}{2 \tau}\right)^{2} .
\end{aligned}
$$

Using $I=\int_{\mathbb{R}^{n}}|\nabla \ln u|^{2} d \mu, \int_{\mathbb{R}^{n}} u d \mu=1$ and Jensen's inequality, we have

$$
\left(I-\frac{n}{2 \tau}\right)^{2}=\left(\int_{\mathbb{R}^{n}}\left(|\nabla \ln u|^{2}-\frac{n}{2 \tau}\right) u\right)^{2} \leq \int_{\mathbb{R}^{n}}\left(|\nabla \ln u|^{2}-\frac{n}{2 \tau}\right)^{2} u d \mu .
$$

This completes the proof of Theorem 2.1

\subsection{Scaling for $\mathrm{W}$}

Consider a standard mollifier $u(x)$ such that $\int_{\mathbb{R}^{n}} u(x) d x=1$ and $u_{\epsilon}(x)=\epsilon^{-n} u\left(\frac{x}{\epsilon}\right), \epsilon>0$. Then, Shannon entropy and Fisher information scale respectively as follows

$$
\begin{aligned}
H\left(u_{\epsilon}\right) & =-\int_{\mathbb{R}^{n}} u_{\epsilon} \ln u_{\epsilon} d \mu \\
& =-\int_{\mathbb{R}^{n}} \epsilon^{-n} u\left(\frac{x}{\epsilon}\right) \ln \left(\epsilon^{-n} u\left(\frac{x}{\epsilon}\right)\right) d \mu \\
& =-\int_{\mathbb{R}^{n}} \epsilon^{-n} u\left(\frac{x}{\epsilon}\right) \ln u\left(\frac{x}{\epsilon}\right)-\int_{\mathbb{R}^{n}} \epsilon^{-n} u\left(\frac{x}{\epsilon}\right) \ln \epsilon^{-n} d \mu \\
& =-\int_{\mathbb{R}^{n}} u(y) \ln u(y) d \mu(y)-\ln \epsilon^{-n} \int_{\mathbb{R}^{n}} u(y) d \mu(y)=H(u)+n \ln \epsilon
\end{aligned}
$$


and

$$
\begin{aligned}
I\left(u_{\epsilon}\right) & =\int_{\mathbb{R}^{n}} \frac{\left|\nabla u_{\epsilon}\right|^{2}}{u_{\epsilon}} d \mu=\int_{\mathbb{R}^{n}} \frac{\left|\nabla \epsilon^{-n} u\left(\frac{x}{\epsilon}\right)\right|^{2}}{\epsilon^{-n} u\left(\frac{x}{\epsilon}\right)} d \mu \\
& =\int_{\mathbb{R}^{n}} \frac{\epsilon^{-2(n+1)}|\nabla u(y)|^{2}}{\epsilon^{-n} u(y)} d \mu=\epsilon^{-2} \int_{\mathbb{R}^{n}} \frac{|\nabla u(y)|^{2}}{u(y)} d \mu=\epsilon^{-2} I(u) .
\end{aligned}
$$

It was already shown that entropy functional $W$ relates well with Shannon entropy and Fisher information, but the scaling done above indicates that both $H(u)$ and $I(u)$ are not scale invariant with respect to dilation factor $u(u) \rightarrow u_{\epsilon}(x)=\epsilon^{-n} u\left(\frac{x}{\epsilon}\right)$. Hence, the need to establish the scale invariance for $W$. Moreover, the decreasing in time monotonicity depends on scale invariance with respect to the dilation factor. Recall

$$
W=\int_{\mathbb{R}^{n}}\left(\tau \frac{|\nabla u|^{2}}{u^{2}}-\ln u\right) u d \mu-\frac{n}{2} \ln (4 \pi \tau)-n .
$$

Using the scale factor $u(x) \rightarrow u_{\epsilon}(x)=\epsilon^{-n} u\left(\frac{x}{\epsilon}\right)$, we have

$$
\begin{aligned}
W_{\epsilon}=W\left(u_{\epsilon}, \tau\right) & =\int_{\mathbb{R}^{n}}\left(\tau \frac{\left|\nabla u_{\epsilon}\right|^{2}}{u_{\epsilon}^{2}}-\ln u_{\epsilon}\right) u_{\epsilon} d \mu-\frac{n}{2} \ln (4 \pi \tau)-n \\
& =\tau I\left(u_{\epsilon}\right)+H\left(u_{\epsilon}\right)-\frac{n}{2} \ln (4 \pi \tau)-n \\
& =\tau \epsilon^{-2} I+H+n \ln \epsilon-\frac{n}{2} \ln (4 \pi \tau)-n .
\end{aligned}
$$

Choosing $\epsilon^{2}=4 \pi \tau$, we have

$$
W\left(u_{\epsilon}, \tau\right)=\int_{\mathbb{R}^{n}}\left[\frac{1}{4 \pi} \frac{|\nabla v|^{2}}{v^{2}}-\ln v\right] v d \mu(y)-n,
$$

where $u_{\epsilon}(x)=\epsilon^{-n} v\left(\frac{x}{\epsilon}\right)$ with $d x=\epsilon^{n} d y$ and $v(y)=\epsilon^{n} u(\epsilon y)$ have been used in the last equation.

Now, suppose at time $t=0, u_{0}(x)>0$ and $\int_{\mathbb{R}^{n}} u_{0}(x) d x=1$, then by the convolution of the heat kernel, we have

$$
u(t, x)=(4 \pi t)^{-\frac{n}{2}} \int_{\mathbb{R}^{n}} e^{-\frac{|x-z|^{2}}{4 t}} u_{0}(z) d z
$$

and

$$
\begin{aligned}
v(t, y)=\epsilon^{n} u(t, \epsilon y) & =\epsilon^{n}(4 \pi t)^{-\frac{n}{2}} \int_{\mathbb{R}^{n}} e^{-\frac{|\epsilon y-z|^{2}}{4 t}} u_{0}(z) d z \\
& =(4 \pi t)^{\frac{n}{2}}(4 \pi t)^{-\frac{n}{2}} \int_{\mathbb{R}^{n}} e^{-\frac{\epsilon^{2}}{4 t}|y-z / \epsilon|^{2}} u_{0}(z) d z \\
& =\int_{\mathbb{R}^{n}} e^{-\pi|y-z / \epsilon|^{2}} u_{0}(z) d z .
\end{aligned}
$$

Since $z \mid \epsilon \rightarrow 0$ as $t \rightarrow \infty$, then $v(y) \rightarrow e^{-\pi|y|^{2}}$ pointwisely for fixed $y$ and the $W$-entropy can be written as

$$
W=\int_{\mathbb{R}^{n}}\left(\frac{1}{4 \pi} \frac{|\nabla v(y)|^{2}}{v(y)^{2}}-\ln v(y)\right) v(y) d \mu(y)-n .
$$

It is also clear that $\nabla v(y) \rightarrow \nabla e^{-\pi|y|^{2}}=-2 \pi y e^{-\pi|y|^{2}}$ and $\ln v(y) \rightarrow \ln e^{-\pi|y|^{2}}=-\pi|y|^{2}$ as $t \rightarrow \infty$. Therefore, by application of dominated convergence theorem as $t \rightarrow \infty$

$$
W \rightarrow \int_{\mathbb{R}^{n}} 2 \pi|y|^{2} e^{-\pi|y|^{2}} d \mu(y)-n=2 \pi \int_{\mathbb{R}^{n}}|y|^{2} e^{-\pi|y|^{2}} d \mu(y)-n=0 .
$$

Combining the above with monotone decreasing property of $W$, we can conclude that

$$
0=\lim _{t \rightarrow \infty} W \tau(t), f(t) \leq W(\tau(t), f(t)) .
$$

This then implies that $W(f, \tau) \geq 0$ for all $t \in(0, \infty)$.

\subsection{Log-Sobolev Inequality}

Note that Log-Sobolev inequality (2.4) is scale invariant, that is, the inequality is preserved under multiplication by a positive constant. Once $W \geq 0$, one can then write

$$
\tau \int_{\mathbb{R}^{n}} \frac{|\nabla u|^{2}}{u} d \mu \geq \int_{\mathbb{R}^{n}} u \ln u d \mu+\frac{n}{2} \ln (4 \pi \tau)+n
$$


which is essentially the Log-Sobolev inequality. Choosing a standard notation $\phi=\sqrt{u}$ with $\int_{\mathbb{R}^{n}} \phi^{2} d \mu=1$, it is then obtained at once

$$
4 \tau \int_{\mathbb{R}^{n}}|\nabla \phi|^{2} d \mu \geq \int_{\mathbb{R}^{n}} \phi^{2} \ln \phi^{2} d \mu+\frac{n}{2} \ln (4 \pi \tau)+n,
$$

which implies

$$
\int_{\mathbb{R}^{n}} \phi^{2} \ln \phi^{2} d \mu \leq \frac{\epsilon^{2}}{\pi} \int_{\mathbb{R}^{n}}|\nabla \phi|^{2} d \mu+C(\epsilon, n) .
$$

The above argument proves Theorem 2.2

\section{Monotonicity of $\Phi(t)$ and the proof of Theorem 2.3}

The aim of this section is to prove the inequality in (2.6). Here, the fundamental solution of the heat equation is used. Let $f(t, x)=P_{t} f(x)$ solve the heat equation, where $P_{t}$ is a one-parameter heat diffusion semigroup generated by $\Delta$.

Setting $v:=\log f(x)$ at $t=0$, we have the initial value problem (from the heat equation)

$$
\left\{\begin{array}{l}
\frac{\partial v}{\partial t}=\Delta v+|\nabla v|^{2} \\
\left.v\right|_{t=0}=\log f
\end{array}\right.
$$

with the diffusion semigroup $v(t, x)=\log P_{t} f(x)$. Following the idea first introduced in [10] (also used in [6]), a nonlinear heat semigroup can be defined by

$$
f(t, x)=\left(P_{t} f(x)^{2}\right)^{\frac{1}{2}}
$$

to obtain a nonlinear heat flow

$$
\left.\frac{\partial f(t, x)}{\partial t}\right|_{t=0}=\Delta f(x)+\frac{|\nabla f(x)|^{2}}{f(x)} .
$$

Using the transformation of $\mathbb{R}^{n}$ onto $j$ th coordinates, $1 \leq j \leq m$, the $j$ th coordinate nonlinear heat flow is precisely written as

$$
\frac{\partial f_{j}\left(t, x_{j}\right)}{\partial t}=\Delta f_{j}\left(t, x_{j}\right)+\frac{\left|\nabla f_{j}\left(t, x_{j}\right)\right|^{2}}{f_{j}\left(t, x_{j}\right)} .
$$

Now define the functional

$$
\Phi(t)=\int_{\mathbb{R}^{n}} \prod_{j=1}^{m} f_{j}(t, x) d \mu(x)
$$

which is known to be differentiable and smoothly continuous by the smoothing properties of the heat kernel semigroup for all $t>0$.

\subsection{Monotonicity formula for $\Phi(\mathrm{t})$}

Lemma 4.1. Let $v_{j}:[0, \infty) \times \mathbb{R}^{n} \rightarrow \mathbb{R}, \quad 1 \leq j \leq m$ be a nonnegative solution of (4.1). Then $\Phi(t)$ is nondecreasing in time and specifically

$$
\Phi^{\prime}(t)=\frac{1}{2} \int_{\mathbb{R}^{n}} \sum_{l \neq k}\left[\nabla_{l} v_{k}-\nabla_{k} v_{l}\right]^{2} \prod_{j=1}^{m} f_{j}(t, x) d \mu(x) .
$$

Proof. Taking time derivative of $\Phi(t)$ and using (4.2), we have

$$
\begin{aligned}
\Phi^{\prime}(t) & =\frac{d}{d t}\left(\int_{\mathbb{R}^{n}} \prod_{j=1}^{m} f_{j} d \mu(x)\right)=\int_{\mathbb{R}^{n}}\left(\sum_{k=1}^{m} \frac{\partial}{\partial t} f_{k}\right) \prod_{j=1, j \neq k}^{m} f_{j} d \mu(x) \\
& =\int_{\mathbb{R}^{n}} \sum_{k=1}^{m}\left(\Delta f_{k}+\frac{\left|\nabla f_{k}\right|^{2}}{f_{k}}\right) \prod_{j=1, j \neq k}^{m} f_{j} d \mu(x) \\
& =\int_{\mathbb{R}^{n}} \sum_{k=1}^{m}\left(\Delta f_{k}\right) \prod_{j=1, j \neq k}^{m} f_{j} d \mu(x)+\int_{\mathbb{R}^{n}} \sum_{k=1}^{m}\left(\frac{\left|\nabla f_{k}\right|^{2}}{f_{k}}\right) \prod_{j=1, j \neq k}^{m} f_{j} d \mu(x) .
\end{aligned}
$$


Applying integration by parts on the first integral yields

$$
\begin{aligned}
\Phi^{\prime}(t) & =-\int_{\mathbb{R}^{n}} \sum_{k, l=1}^{m}\left(\nabla f_{k}, \nabla f_{l}\right) \prod_{j=1,}^{m} f_{j \neq k, l} d \mu(x)+\int_{\mathbb{R}^{n}} \sum_{k=1}^{m}\left(\frac{\left|\nabla f_{k}\right|^{2}}{f_{k}}\right) \prod_{j=1, j \neq k}^{m} f_{j} d \mu(x) \\
& =-\int_{\mathbb{R}^{n}} \sum_{k, l=1}^{m}\left[\frac{\nabla f_{k}}{f_{k}} \cdot \frac{\nabla f_{l}}{f_{l}}-\frac{\left|\nabla f_{k}\right|^{2}}{f_{k}^{2}}\right] \prod_{j=1}^{m} f_{j} d \mu(x) \\
& =\frac{1}{2} \int_{\mathbb{R}^{n}} \sum_{k, l=1, k \neq l}^{m}\left[\frac{\left|\nabla f_{k}\right|^{2}}{f_{k}^{2}}+\frac{\left|\nabla f_{l}\right|^{2}}{f_{l}^{2}}-2 \frac{\nabla f_{k}}{f_{k}} \cdot \frac{\nabla f_{l}}{f_{l}}\right] \prod_{j=1}^{m} f_{j} d \mu(x) \\
& =\frac{1}{2} \int_{\mathbb{R}^{n}} \sum_{k \neq l}^{m}\left[\frac{\nabla f_{k}}{f_{k}}-\frac{\nabla f_{l}}{f_{l}}\right]^{2} \prod_{j=1}^{m} f_{j} d \mu(x)=\frac{1}{2} \int_{\mathbb{R}^{n}} \sum_{k \neq l}^{m}\left[\nabla v_{k}-\nabla v_{l}\right]^{2} \prod_{j=1}^{m} f_{j} d \mu(x) .
\end{aligned}
$$

There is equality in (4.4) if and only if

$$
\frac{\nabla f_{k}}{f_{k}}-\frac{\nabla f_{l}}{f_{l}}=0 .
$$

Since it is known that each $f_{j}$ is strictly positive and each $v_{k}$ is positive, smooth and bounded for all time $t>0$, we therefore conclude that the quantity $\Phi(t)$ is nondecreasing for all $t>0$.

Proof of Theorem 2.3. For any nonnegative measurable function $f_{j}, 1 \leq j \leq m$, it seen that the functional $\Phi(t)$ in $(4.3)$ is nondecreasing for all $t>0$. Then, by the monotonicity property of $\Phi(t)$, the quantity

$$
\tilde{\Phi}(t)=\int_{\mathbb{R}^{n}} \prod_{j=1}^{m} f_{j}^{\frac{1}{p_{j}}}(t, x) d \mu(x)
$$

is also nondecreasing. Indeed, taking time derivative of $\tilde{\Phi}(t)$ as follows

$$
\tilde{\Phi}^{\prime}(t)=\int_{\mathbb{R}^{n}} \sum_{k=1}^{m} \frac{1}{p_{k} f_{k}}\left(\frac{\partial}{\partial t} f_{k}\right) \prod_{j=1}^{m} f_{j}^{\frac{1}{p_{j}}} d \mu .
$$

Setting $v=\log f$ as before, where $f$ solves the heat equation, then

$$
f_{j}=e^{v_{j}} \forall j, \prod_{j=1}^{m} f_{j}^{\frac{1}{p_{j}}}=e^{\sum_{j=1}^{m} \frac{1}{p_{j}} v_{j}} \text { and } \frac{\partial}{\partial t} f_{k}=\Delta f_{k}=\Delta v_{k}+\left|\nabla v_{k}\right|^{2} f_{k}
$$

Therefore

$$
\begin{aligned}
\tilde{\Phi}^{\prime}(t) & =\int_{\mathbb{R}^{n}}\left[\sum_{k=1}^{m} \frac{1}{p_{k}}\left(\Delta v_{k}+\left|\nabla v_{k}\right|^{2}\right)\right] e^{\sum_{j=1}^{m} \frac{1}{p_{j}} v_{j}} d \mu \\
& =\int_{\mathbb{R}^{n}}\left[-\left|\sum_{k=1}^{m} \frac{1}{p_{k}} \nabla v_{k}\right|^{2}+\sum_{k=1}^{m} \frac{1}{p_{k}}\left|\nabla v_{k}\right|^{2}\right] e^{\sum_{j=1}^{m} \frac{1}{p_{j}} v_{j}}
\end{aligned}
$$

by using integration by parts. Rewriting the first term on the right hand side of the last equation as follows (since $\left.\max _{k} p_{k}^{-1} \leq 1\right)$

$$
\left|\sum_{k=1}^{m} \frac{1}{p_{k}} \nabla v_{k}\right|^{2} \leq \sum_{k=1}^{m} \frac{1}{p_{k}}\left|\nabla v_{k}\right|^{2}
$$

reveals that $\tilde{\Phi}^{\prime}(t) \geq 0$. Therefore

$$
\limsup _{t \rightarrow 0} \tilde{\Phi}(t) \leq \liminf _{t \rightarrow \infty} \tilde{\Phi}(t) .
$$

By Fatou's lemma, we have

$$
\int_{\mathbb{R}^{n}} \prod_{j=1}^{m} f_{j}^{\frac{1}{p_{j}}}(x) \leq \limsup _{t \rightarrow 0^{+}} \tilde{\Phi}(t) .
$$

Indeed, there is equality in (4.5) since $\lim _{t \rightarrow 0} P_{t} f=f$. It then suffices to prove that

$$
\liminf _{t \rightarrow \infty} \tilde{\Phi}(t) \leq \prod_{j=1}^{m}\left(\int_{\mathbb{R}^{n}} f_{j}\right)^{\frac{1}{p_{j}}} .
$$


The proof of (4.6) can be made more rigorous but outline is given here. Now, observe that $f_{j}(t, x)$ depends on $x_{j}$ coordinate not on $x$ itself, then we have

$$
f_{j}\left(t, x_{j}\right)=(4 \pi t)^{-\frac{n}{2}} \int_{\mathbb{R}^{n_{j}}} e^{-\left|x_{j}-z\right|^{2} / 4 t} f_{j}(z) d \mu(z) .
$$

Notice that each $f_{j}$ above solves the heat equation with initial condition $f_{j}(0, x)=f_{j}(x)$. It is then written

$$
\tilde{\Phi}(t)=(4 \pi t)^{-\frac{\sum_{j=1}^{m} \frac{n}{p_{j}}}{2}} \int_{\mathbb{R}^{n}} \prod_{j=1}^{m}\left(\int_{\mathbb{R}^{n}} e^{-|| x_{j}-z \|^{2} / 4 t} f_{j}(z) d \mu(z)\right)^{\frac{1}{p_{j}}} d \mu(x) .
$$

Noting also that $\sum_{j=1}^{m} \frac{n}{p_{j}}=n$. By rescaling argument, using $u_{\epsilon}(x) \longrightarrow \epsilon^{-n} v\left(\frac{x}{\epsilon}\right), \epsilon>0$, (i.e., by making the change of variables $x=\epsilon y, \quad d x=\epsilon d y$ ), we then have the transformation

$$
\tilde{\Phi}(t)=\frac{\epsilon^{n}}{(4 \pi t)^{\frac{n}{2}}} \int_{\mathbb{R}^{n}} \prod_{j=1}^{m}\left(\int_{\mathbb{R}^{n}} e^{-\frac{\epsilon^{2}}{4 t}\left\|y_{j}-z / \epsilon\right\|^{2}} f_{j}(z) d \mu(z)\right)^{\frac{1}{p_{j}}} d \mu(y) .
$$

Choosing a scaling factor $\epsilon^{2}=4 \pi t$, by convolution property and Fubini's theorem yield

$$
\liminf _{t \rightarrow \infty} \tilde{\Phi}(t) \leq \prod_{j=1}^{m}\left(\int_{\mathbb{R}^{n}}\left|f_{j}(z)\right| d \mu(z)\right)^{\frac{1}{p_{j}}} \int_{\mathbb{R}^{n}}\left(e^{-\pi\|y\|^{2}}\right)^{\frac{1}{p_{j}}} d \mu(y) .
$$

The claim (4.6) then follows immediately, since $\sum_{j=1}^{m} \frac{1}{p_{j}}=1$ by the hypothesis of the theorem and $\int_{\mathbb{R}^{n}} e^{-\pi\|y\|^{2}} d \mu(y)=1$ by standard Gauss integral.

\section{Conclusion}

This paper constructed two entropy monotonicity formulas through the heat flows. The entropy formulas are employed to derive some functional and geometric inequalities. Specifically, it was shown that the first entropy monotonicity formula constructed is intimately related to the concavity of the power of Shannon entropy and Fisher Information, from which the associated logarithmic Sobolev inequality for probability measure is recovered. The second monotonicity formula constructed, with convolution and diffusion semigroup properties of the heat kernel, was used to establish the proof of the multilinear Hölder inequalities.

This study discovered that many functional and geometric inequalities can be retrieved as consequences of monotonicity properties of heat flow entropies. This approach is more simplified than existing methods like rearrangement or using either heat flow or entropy formula independently. This study will help analysts and mathematics users to uncover many other important consequences of heat flow monotonicity. Thus a new theory on this subject may be arrived at.

A major challenge with the heat flow is that the heat equation needs to depend on all of the coordinates, i.e., the $j$ thcoordinate heat flow wants to depend on $x_{k}$ for all $k$. This poses some sort of difficulty in extending the method used in this paper to Riemannian settting. An attempt to circumvent this is desirable.

\section{Acknowledgment}

The authors wish to thank the anonymous referees for their useful comments. A gratefully acknowledges his PhD supervisor, Prof. Ali Taheri of Sussex University, who first suggested this subject few years ago and for insightful advices he made then. This research is supported by Landmark University, Nigeria.

\section{Supplementary material}

Supplementary material associated with this article can be found, in the online version, at doi:10.1016/j.amc.2019.124640.

\section{References}

[1] A. Abolarinwa, Heat kernel estimates and asymptotic of w-entropy on stochastically complete Riemannian manifolds, J. Nonlinear Funct. Anal. 2017 (2017) 1-14. Article ID 51.

[2] A. Abolarinwa, Differential Harnack and logarithmic Sobolev inequalities along Ricci-harmonic map flow, Pacific J. Math. 278 (2) (2015) 257-290.

[3] A. Abolarinwa, N. Oladejo, S. Salawu, On the entropy formulas and solitons for the Ricci-harmonic flow, Bull. Iran. Math. Soc. (2019), doi:10.1007/ s41980-018-00192-1.

[4] J. Bennet, Heat-flow monotonicity related to some inequalities in euclidean analysis, Contemp. Math. 505 (2010) 85-96.

[5] J. Bennett, N. Bez, Generating monotone quantities for the heat equation, J. Reine Angew. Math. (2017).

[6] J. Bennet, A. Carbery, M. Christ, T. Tao, The Brascamp-Lieb inequalities: finiteness, structure and extremals, Preprint:math.MG/0505065.

[7] J. Bennet, A. Carbery, M. Christ, T. Tao, Finite bounds for Hölders-Brascamp-Lieb multilinear inequalities,. Preprint:math.MG/0505691.

[8] N.M. Blachman, The convolution inequality for entropy powers, IEEE Trans. Inform. Theory 2 (1965) $267-271$.

[9] V.I. Bogachev, Gaussian measures, AMS (1998).

[10] E.A. Carlen, E.H. Lieb, M. Loss, A sharp analog of Young's inequality on $s^{n}$ and related entropy inequalities, J. Geom. Anal. 14 (3) (2004) 487-520.

[11] T.H. Colding, W.P. Minicozzi II, Monotonicity and its analytic and geometric implications, Proc. Natl. Acad. Sci. USA 110 (2012) $19233-19236$. 
[12] M.H.M. Costa, A new entropy power inequality, IEEE Trans. Inf. Theory IT-31 (6) (1985) 751-760.

[13] A. Grigorýan, The heat equation on non-compact Riemannian manifolds, (in Russian) Matem Sbornik 182 (1) (1991) 55-87. Translation in Maths (1992).

[14] A. Grigorýan, Gaussian upper bounds for the heat kernel on arbitrary manifold, J. Dif. Geom. 45 (1) (1997) $33-35$.

[15] A.G. ýan, Heat kernel and analysis on manifolds, AMS (2009).

[16] L. Gross, Logarithmic Sobolev inequalities, Am. J. Math 97 (1) (1975) 1061-1083.

[17] L. Gross, Logarithmic Sobolev inequalities and contractivity properties of semigroups, in: Dirichlet forms (Varenna) Lecture Notes in Math., 1992.1563.

[18] M. Ledoux, Heat flows, geometric and functional inequalities, in: Proceedings of the International Congress of Mathematicians. Vol. IV: Invited Lectures (Seoul), Kyung Moon, Seoul, 2014, pp. 117-135.

[19] P. Li, S.T. Yau, On the parabolic kernel of the Schrödinger equation, Acta Math. 156 (1986) 139-168.

[20] L.H. Loomis, H. Whitney, An inequality related to the isoperimetric inequality, Bull. Am. Math. Soc. 55 (1949) $961-962$.

[21] L. Ni, The entropy formula for linear heat equation, J. Geom. Anal. 14 (2) (2004) 85-98

[22] G. Perelman, The entropy formula for the Ricci flow and its geometric application, arXiv:math.DG/0211159v1 (2002).

[23] C.E. Shannon, A mathematical theory of communication, Bell Syst. Technol. J. 27 (1948) 379-423.

[24] A.J. Stam, Some inequalities satisfied by the quantities of information of fisher and Shannon, Inf. Control 2 (1959) 101-112.

[25] G. Toscani, Lyapunov functionals for the heat equation and sharp inequalities, Atti della Accad. Pelorit. Pericol. 91 (1) (2013).

[26] G. Toscani, An information-theoretic proof of Nash's inequality, Rend. Lincei Mat. Appl. 24 (2013) 83-93.

[27] C. Villani, A short proof of the concavity of entropy power, IEEE Trans. Info. Theory 46 (4) (2000) 1695-1696.

[28] Q. Zhang, Sobolev Inequalities, Heat Kernels under Ricci Flow, and the Poincare Conjecture, CPC Press, 2011. 